\title{
Distribution and potential impacts of non-native Chinese pond mussels Sinanodonta woodiana (Lea, 1834) in Bavaria, Germany
}

\author{
Andreas H. Dobler $\mathbb{D} \cdot$ Philipp Hoos $(\mathbb{D} \cdot$ \\ Juergen Geist 1
}

Received: 1 September 2021 / Accepted: 18 January 2022 / Published online: 15 February 2022

(C) The Author(s) 2022

\begin{abstract}
The Chinese pond mussel, Sinanodonta woodiana, is non-native to European freshwater systems. Originating from Asia, it was brought to Europe in the 1970s and is now spreading across many countries. Management of the species is currently limited by a lack of information on the actual distribution and population characterisation of $S$. woodiana as well as on the co-occurrence of native mussels potentially resulting in competition. For this paper, we examined nine water systems in the German federal state of Bavaria with randomly observed and anecdotally reported $S$. woodiana occurrences. We recorded the density and biomass of $S$. woodiana and of the co-existing mussel species as well as the habitat characteristics. We found $S$. woodiana in eight water systems, co-existing with other native and invasive mussel species in seven of them. The distribution of $S$. woodiana was geographically widespread throughout Bavaria, indicating great invasion potential. In one fish pond, $S$. woodiana was the only mussel species occurring with the highest mean biomass $\left(1286.0 \pm 1067.7 \mathrm{~g} \mathrm{~m}^{-2}\right)$ and with the biggest specimen $(25.0 \mathrm{~cm}$ and $1616.0 \mathrm{~g})$. The wide range of habitat variables matched the expectation of a wide ecological niche for the species. This study provides
\end{abstract}

A. H. Dobler $\cdot$ P. Hoos $\cdot$ J. Geist $(\bowtie)$

Aquatic Systems Biology Unit, Department of Life

Science Systems, Technical University of Munich,

Muehlenweg 22, 85354 Freising, Germany

e-mail: geist@wzw.tum.de evidence that $S$. woodiana has already become established in many water systems in Bavaria, with fish ponds and fisheries management practises being a likely vector. To counteract this species and to conserve native mussel species, it is important to implement effective legislation, to take measures to eradicate this invasive mussel transnationally, and to raise public awareness.

Keywords Chinese pond mussel $\cdot$ Sinanodonta woodiana - Unionidae · Freshwater mussels · Invasive species $\cdot$ Co-occurrence

\section{Introduction}

Biological invasions can have major impacts on local freshwater ecosystems (Keller et al. 2011; Malmqvist and Rundle 2002; Strayer and Dudgeon 2010). Thus, the introduction of alien species can bring about an increase of competition in several contexts (e.g. food resources, reproduction) as well as predation which could result in a decline of native species (Sousa et al. 2014; Zahner-Meike and Hanson 2001). Native freshwater mussel populations have declined globally in recent decades and most of them are now considered endangered (Lopes-Lima et al. 2018). These declines result mainly from water pollution, habitat degradation and fragmentation as well as climate change (Dudgeon et al. 2006; Lydeard et al. 2004; Regnier et al. 2009; Stoeckl et al. 2020; Strayer et al. 2004; 
Young and Williams 1983). In addition, the negative effects of invasive alien species (IAS) can be important. For instance, predation of invasive muskrat (Ondatra zibethicus) can cause considerable damages to mussel populations (Zahner-Meike and Hanson 2001) and also invasive freshwater crayfish appear to have negative impacts on native mussel populations (Dobler and Geist 2022; Meira et al. 2019; Schmidt and Vandré 2012; Sousa et al. 2019). Also, the spread of invasive freshwater bivalves can lead to increasing competition pressures (Urbańska et al. 2021). The relatively small invasive Asian clam Corbicula fluminea (Müller, 1774), which, however, occurs in high densities, and the invasive zebra mussel, Dreissena polymorpha (Pallas, 1771), both compete strongly with native mussels for food as a result of their high filtration rates (Cohen et al. 1984; Phelps 1994; Strayer et al. 1999). In Europe, both species are well established in many standing or slow flowing water bodies (see Karatayev et al. 2005; Van der Velde et al. 2010). Due to its ability to attach to solid objects using byssus threads, D. polymorpha can overgrow native bivalves, which can negatively affect the physiological condition of and also deform or at least overturn and thus kill these mussels (Ożgo et al. 2020; Sousa et al. 2011). Aside from this, the direct impact of dreissenid fouling on industrial and infrastructural facilities results in manifest technical and financial damage (Cuthbert et al. 2021; Sousa et al. 2014), which attracts widespread attention in society. In contrast, the invasion of unionids has remained relatively unnoticed and the potential effects have not yet been considered. Nevertheless, there is increasing evidence that another occurring non-native freshwater mussel, the Chinese pond mussel Sinanodonta woodiana (Lea, 1834), has competitive advantages over other unionids native to Europe. For example, this species can use a broader range of suitable fish as hosts, achieves higher infestation rates and develops faster than native mussel species (Douda et al. 2012; Huber and Geist 2019). In addition, the quantity of glochidia is larger in $S$. woodiana compared to native unionids, breeding is not limited to one single action per year (Labecka and Czarnoleski 2019; Labecka and Domagala 2018) and the glochidia are also more persistent over a range of temperatures (Benedict and Geist 2021). All this leads to increased reproduction success for $S$. woodiana. Conversely, the recruitment of native unionids could be reduced if a host fish has already been infested with glochidia of $S$. woodiana as has been shown by Donrovich et al. (2017) for Anodonta anatina. In addition, adult specimens have been reported to tolerate a wider range of substratum conditions in comparison with native mussel species (Urbańska et al. 2021).

$S$. woodiana originates from the Yangtze River basin and from Taiwan (see Kondakov et al. 2018; Lopes-Lima et al. 2020) and is spreading to many countries globally (see e.g. Bespalaya et al. 2018 for Siberia; Bogan et al. 2011 for USA; Kondakov et al. 2018 for Uzbekistan; Konecny et al. 2018 for Europe; Kondakov et al. 2020 for Russia; Bolotov et al. 2016; Zieritz et al. 2018a for Malaysia and Indonesia; Zieritz et al. 2018b for Borneo; Zieritz et al. 2016 for Malaysia). In Europe, this species was first recorded in western Romania in 1979 (Sarkany-Kiss 1986) and then three years later near Arles in southern France (Adam 2010). Initially, its spread was slow and restricted to artificial heated water (see Urbańska et al. 2012) but has become faster in the last two decades (Lopes-Lima et al. 2017). Konecny et al. (2018) described the initial introduction of this species to Europe as resulting from a commercial import of Asian carp from the Yangtze River basin to hatcheries in Romania in the early 1960s, while a further spread of $S$. woodiana from the Amur River basin that were brought to hatcheries in Hungary could not be genetically verified in Europe. With its ability to adapt to cold water, this mussel species has succeeded in invading many water systems within Europe (Konecny et al. 2018). In addition, this species is sold in many outlets such as garden centres and construction markets that provide pond products as well as in aquaristics online stores, often erroneously named as "European pond mussel" (pers. obs.). This provides S. woodiana with an additional opportunity to spread even further.

Despite the situation that $S$. woodiana has colonised many European countries, the European Union lacks a consistent approach for classification and management. The German legal status, for example, classifies $S$. woodiana as 'potentially invasive' (Rabitsch and Nehring 2017) because of the lack of documentation on the actual distribution and invasiveness of $S$. woodiana. This shows the need to evaluate the actual distribution of $S$. woodiana as well as its co-occurrence with native mussel species. For effective management of $S$. woodiana, 
systematic documentation of its habitat preferences, distribution pathways and its effects on native mussels in the event of coexistence would provide crucial information. Some studies already exist that document single populations or the spatial distribution of $S$. woodiana, partly combined with an analysis of the co-occurrence and competition with native mussels, while other studies are focussed on single populations and the description of their habitat conditions. In this study, we have systematically investigated the habitat conditions and mussel community of various water bodies over a larger regional scale.

Therefore, we monitored nine water systems with randomly observed and anecdotally reported $S$. woodiana occurrences in Bavaria, Germany. Our main aims were (i) to verify the alleged occurrences of $S$. woodiana in Bavaria as well as to assess establishment of this species in these water systems; (ii) to characterise the habitat conditions where the species is found; (iii) to characterise the $S$. woodiana populations with respect to population density, mussel length and biomass and (iv) to assess the co-occurrence and potential interactions with native mussel species. We hypothesised that (i) S. woodiana still only occurs sporadically in the study region, (ii) the distribution of $S$. woodiana is limited to artificial or anthropogenic water bodies like fish ponds and (iii) the density and biomass of $S$. woodiana is higher than that of native mussel species.

\section{Methods}

\section{Study area}

The study area covers the federal state of Bavaria in Germany with its three main catchment areas of the Danube, Main and Elbe. We searched for references of known $S$. woodiana occurrences in the federal species protection database (LfU 2020) as well as for hints from the public collected by the Bavarian coordination office for freshwater mussel conservation (personal communication) over a period of four years. We systematically examined nine water bodies (Fig. 1) with a speculated occurrence of $S$. woodiana. All necessary permissions for this study including protected species and protected areas were obtained (reference numbers: RMF-SG55.1-8646-7-111-2 for Middle Franconia, 55.3-8646-2/856 for Swabia,
ROP-SG55.1-8622.1-47-2-3 for Upper Palatinate and 41.0.03/8231 for the administrative district of Passau).

\section{Data collection}

Based on the different macrohabitats of each water body, we haphazardly distributed a minimum of six sampling plots around the area of the suspected $S$. woodiana occurrence by randomly placing sampling frames onto the water bottom. We used $0.5 \times 0.5 \mathrm{~m}$ or $1 \times 1 \mathrm{~m}$ frames respectively a twelvemetre-long and at both ends connected chain with marks all $3 \mathrm{~m}$ defining the 4 corners $\left(=9 \mathrm{~m}^{2}\right)$ to define the spots. This resulted in a total of 129 quadrats of $1 \mathrm{~m}^{2}$ (i.e. 0 to 50 per site) and 19 quadrats of $9 \mathrm{~m}^{2}$ (i.e. 0 to 12 per site). We systematically started with the smallest frame before switching to the next largest frame after 5 spots with no mussels to ensure sufficient quantity. At sampling spots with a water depth of more than $1 \mathrm{~m}$ or with high turbidity, sampling was performed by scuba diving.

Abiotic habitat parameters

To characterise the habitats and to determine the ecological tolerance of $S$. woodiana, we measured temperature $\left(\mathrm{T}\right.$, in $\left.{ }^{\circ} \mathrm{C}\right)$, dissolved oxygen $\left(\mathrm{O}_{2}\right.$, in $\mathrm{mg} \mathrm{L}^{-1}$ ), $\mathrm{pH}$-value, and electric conductivity (Cond., in $\mu \mathrm{S} \mathrm{cm}^{-1}$, relative to $25^{\circ} \mathrm{C}$ ) using a handheld Multi 3630 IDS F meter (WTW, Weilheim, Germany) once in the open water (FW) as well as in a sample of interstitial water taken at a substratum depth of $10 \mathrm{~cm}$ (IN) in the middle of the sampling spot (Geist and Auerswald 2007). We also measured the turbidity (Turb, in NTU) using a handheld Turb® $355 \mathrm{~T}$ (WTW, Weilheim, Germany). At spots we had to sample by scuba diving, we took samples of interstitial water as well as of open water above the ground and immediately delivered these samples to the surface for measurements.

To characterise the substrate, we measured the penetration resistance ( $\mathrm{PR}$, in $\mathrm{kg} \mathrm{cm}^{-2}$ ) using a handheld penetrometer (Eijkelkamp Agrisearch Equipment, Giesbeek, The Netherlands) as well as the redox potential $(\mathrm{Eh}$, in $\mathrm{mV})$ in situ in the open water and in the interstitial water (10 cm depth) as per Geist and Auerswald (2007) using a handheld pH 3110 meter (WTW, Weilheim, Germany) together 
Fig. 1 Map of the study area with the three main drainage systems of Bavaria, Germany. Black crosses indicate the nine sampling locations

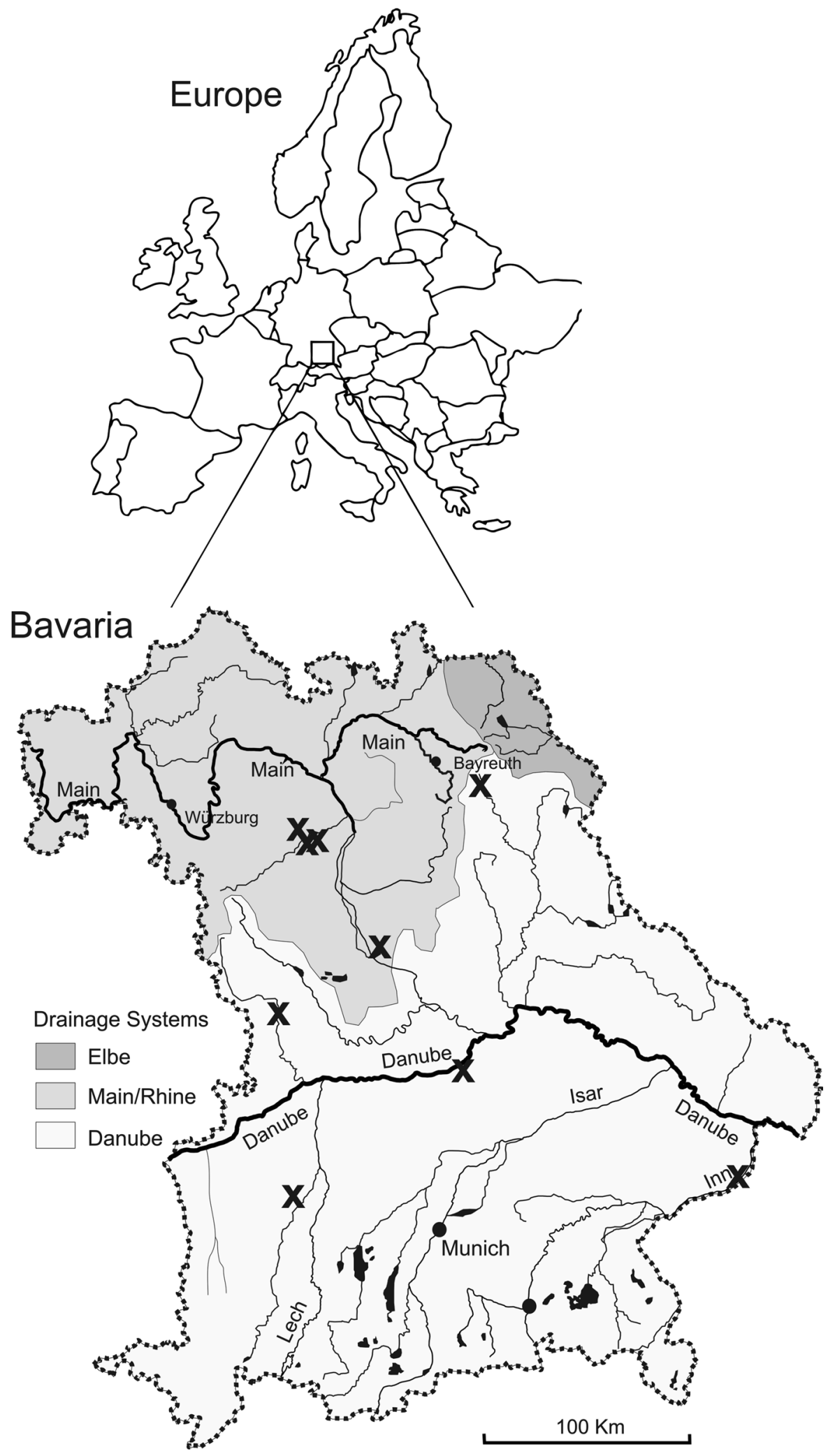


with a platinum electrode and an $\mathrm{Ag} / \mathrm{AgCl}_{2}$-reference electrode. At scuba diving spots the interstitial redox measurement was performed within the interstitial water samples. In addition, the water depth and, if present, the thickness of the silt layer were measured using a measuring rod $( \pm 0.5 \mathrm{~cm})$. For diving spots the water depth was measured using a depth gauge $( \pm 0.25 \mathrm{~m})$.

In running waters, we measured the width of the waterbody using a measuring tape $( \pm 0.05 \mathrm{~m})$ for widths up to $25 \mathrm{~m}$ and the measuring tool of a satellite image viewer (https://geoportal.bayern.de/bayer natlas) for widths over $25 \mathrm{~m}$. In accordance with common techniques in hydrological studies, we measured the flow velocities $\left(\mathrm{v}\right.$, in $\left.\mathrm{m} \mathrm{s}^{-1}\right)$ at $60 \%$ depth of the water column $(\mathrm{mc})$ as well as at $2 \mathrm{~cm}$ below the surface (su) (see Stoeckl and Geist 2016), using a handheld flowmeter (Flowtherm NT, Höntzsch, Waiblingen, Germany). Since the pond at Muenchsmuenster was drained at the time of sampling, no abiotic water parameters could be collected.

\section{Biotic parameters}

We collected all mussel specimens that could be detected visibly or by touch within the defined spots. After the abiotic samples, we also disturbed the sediment by hand to a maximum of $50 \mathrm{~cm}$ depth if possible, e.g. in fine sediment, to find buried individuals. All collected mussels were counted and determined at the species level. The wet body mass of each unionid specimen was weighed separately using field scales $( \pm 1 \mathrm{~g})$ and its shell length was measured using callipers $( \pm 0.1 \mathrm{~mm})$. Mussels with attached dreissenids were cleaned before the measurements and the wet body mass of the attached Dreissena spp. specimens were weighed separately. All Corbicula spp. specimens found were counted and weighed together. All protected mussels were immediately returned to the sample location, whereas invasive mussels were not released.

The coverage of the macrophytes (Marcoph., in \%) within the sampling frames was documented.

\section{Statistical analyses}

We summarised all found mussels and calculated the mussel density (in Ind $\mathrm{m}^{-2}$ ) and biomass (in $\mathrm{g} \mathrm{m}^{-2}$ ) in total and for each mussel species separately for each water system. Due to the high amount of dreissenid bundles in the Rothsee consisting mainly of dead shells as well as live specimens it was not practicable to examine each single mussel specimen. Therefore, we did not include dreissenids in density and biomass calculations. We also calculated the average shell length and mean wet weight of $S$. woodiana for each water body. For habitat parameters, maximum, minimum and mean values were calculated. Unless stated otherwise, arithmetic mean and standard deviation (SD) were calculated and reported as mean \pm standard deviation. We computed a generalised linear model (GLM; model 1) using the "glm"-function within R (version 4.1.0; R Core Team 2020) with a binary value of plots with (1) and without (0) mussel occurrence as the response variable and with the abiotic and biotic values $\left(\mathrm{O}_{2} \mathrm{FW}, \mathrm{O}_{2} \mathrm{IN}, \mathrm{T} \mathrm{FW}\right.$, T IN, Lf FW, Lf IN, pH FW, pH IN, v su, v mc, water depth, Macroph., Turb, PR, Eh FW, Eh IN) as predictors. We used the function "stepAIC" to select the best model and set the significance levels to $\mathrm{p}<0.05$.

To determine the proportion of explained variation in the abundance and biomass data of the native mussel species by physico-chemical parameters as well as the abundance or biomass of $S$. woodiana, non-parametric distance-based linear modelling (DistLM) using PRIMER (version 7) with the PERMANOVA + add on (Plymouth Marine Laboratory, Plymouth, UK; Anderson et al. 2008) was carried out with 999 permutations. The model was based on the Bray-Curtis distance resemblance. AIC (Akaike Information Criterion) was used as the selection criterion and the procedure of selection followed a BEST analysis. To visualise the community structure and the similarity of the different spots, we plotted the DistLM in a distance-based redundancy analysis (dbRDA) for the native mussel density and biomass with bubbles representing the species contributions. We only used environmental variables with a significant contribution to the relationship with the abundance or biomass data of the native mussel species as well as abundance or biomass of $S$. woodiana as an overlay. 


\section{Results}

\section{Population characteristics}

We sampled a total of 148 plots with a total of $300 \mathrm{~m}^{2}\left(129 \times 1 \mathrm{~m}^{2}\right.$ and $\left.19 \times 9 \mathrm{~m}^{2}\right)$ in nine different water systems across Bavaria and found mussels in $55.4 \%$ of the samples. We could verify the establishment of $S$. woodiana in eight of the nine sampled water systems where it was expected to occur based on random observations and anecdotal reporting. Within $35.1 \%$ of our sampling plots we found 100 individuals of $S$. woodiana. In addition, we could find another 92 individuals outside of our plots. We found the native mussel species A. anatina, A. cygnea and $U$. pictorum in five, $U$. tumidus in one and the other invasive mussel species C. fluminea and Dreissena spp. also in one of the nine sampled water bodies. Over all samples including spots without mussels, the mean mussel density was $2.2 \pm 4.4 \mathrm{Ind} \mathrm{m}^{-2}$ with a mean biomass of $35.9 \pm 191.0 \mathrm{~g} \mathrm{~m}^{-2}$. For $S$. woodi-

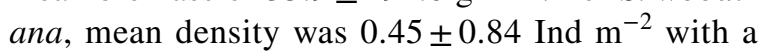
mean biomass of $150.8 \pm 427.2 \mathrm{~g} \mathrm{~m}^{-2}$. We found
Fig. 2 Percentage contribution of (a) the number of individuals and (b) the biomass of all native and invasive mussel species for each sampled water body

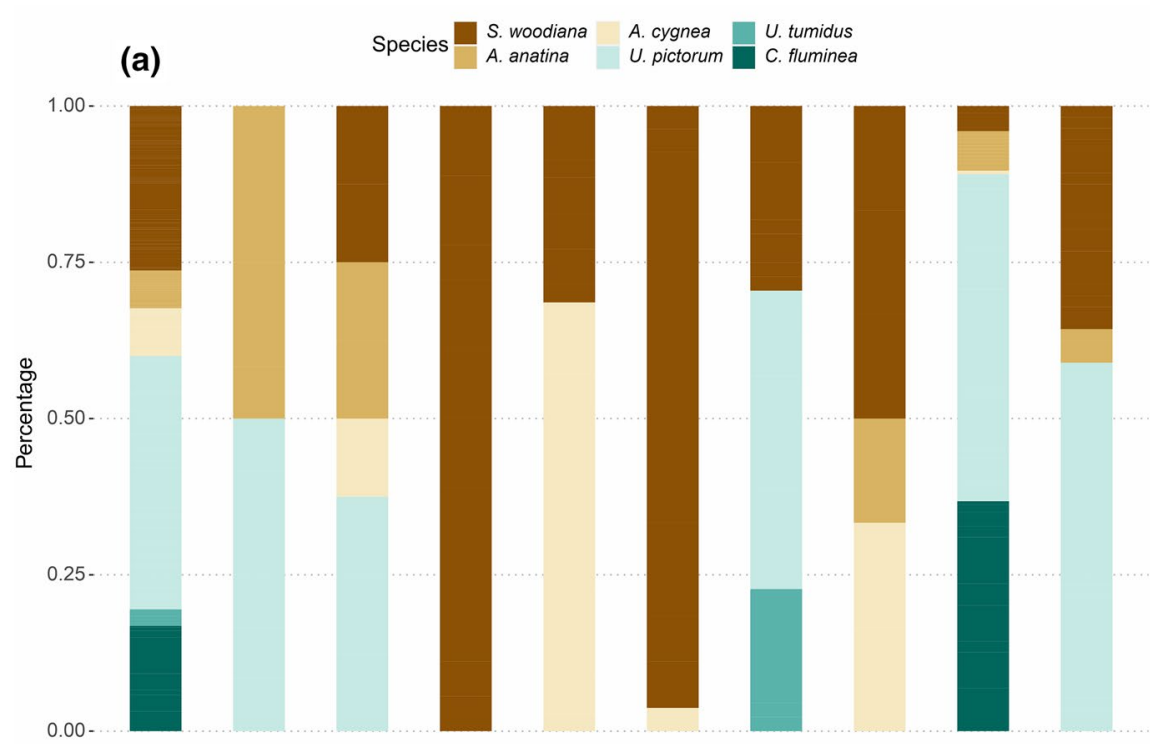

(b)

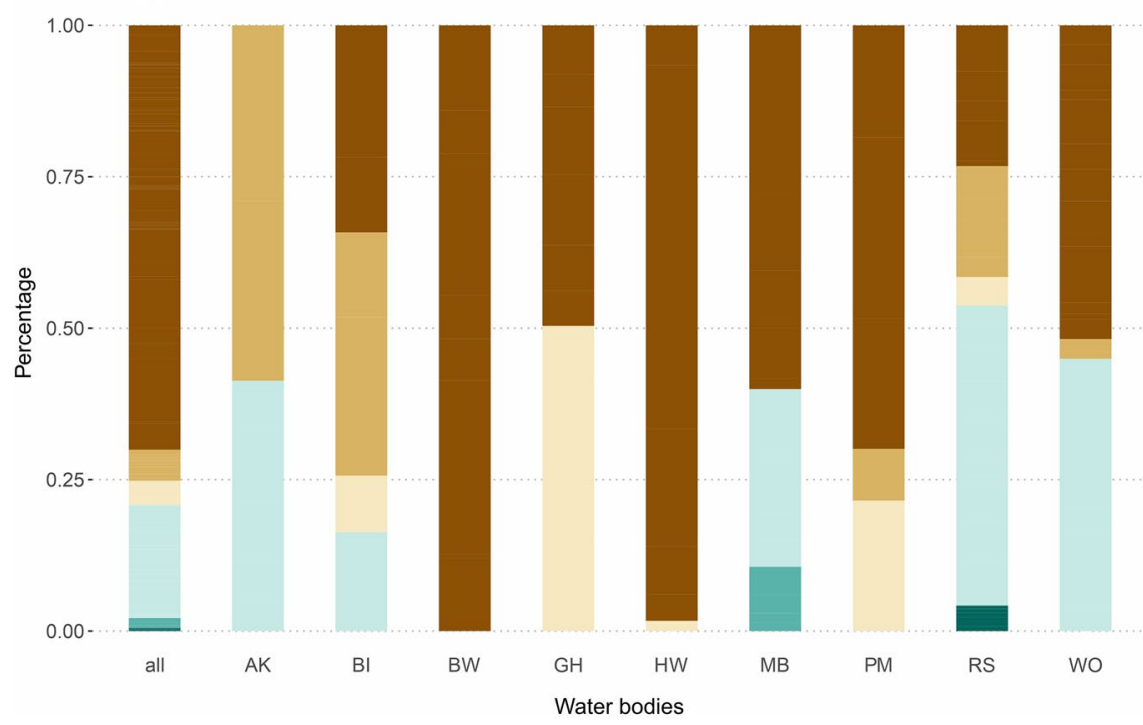


the highest mussel density in the reservoir Rothsee with $5.8 \pm 7.1$ Ind $\mathrm{m}^{-2}$ and a maximum of 26 Ind $\mathrm{m}^{-2}$. This reservoir also had the highest mussel diversity with a percentage contribution of S. woodiana, A. anatina, A. cygnea, U. pictorum and $C$. fluminea with $4.0 \%, 6.3 \%, 0.1 \%, 52.3 \%$ and $36.8 \%$ in number of individuals (see Fig. 2a) and with $23.2 \%, 18.3 \%, 4.7 \%, 49.5 \%$ and $4.2 \%$ in biomass (see Fig. 2b), respectively. In the fish pond Burlafinger Weiher, S. woodiana was the only mussel species occurring and had the highest mean biomass $\left(1286.0 \pm 1067.7 \mathrm{~g} \mathrm{~m}^{-2}\right)$ of all sampled water systems. We also found the largest specimen in this pond at $25.0 \mathrm{~cm}$ and $1616.0 \mathrm{~g}$ (Fig. 3).

In seven of the nine sampled water systems and in $14.2 \%$ of all sampling spots, $S$. woodiana was found co-occurring with native (A. anatina,
A. cygnea, U. pictorum, U. tumidus) or other invasive mussel species (C. fluminea, D. bugensis and D. polymorpha). The reservoir Rothsee, which is connected to the Main-Danube-channel and serves as a water compensation body, hosts most of the species mentioned above excluding $U$. tumidus.

\section{Habitat characterisation}

We additionally characterised the habitat by measuring abiotic and biotic variables in 95 sampling spots. We found mussels in 76 of these sampling spots whereas in 19 spots mussels could not be detected. A significant difference of plots with and without mussels could only be detected in dissolved oxygen of the water column (ANOVA; $F=6.223 ; p<0.05$ ) with $10.2 \pm 2.4 \mathrm{mg} \mathrm{L}^{-1}$ and $8.6 \pm 1.0 \mathrm{mg} \mathrm{L}^{-1}$, respectively.
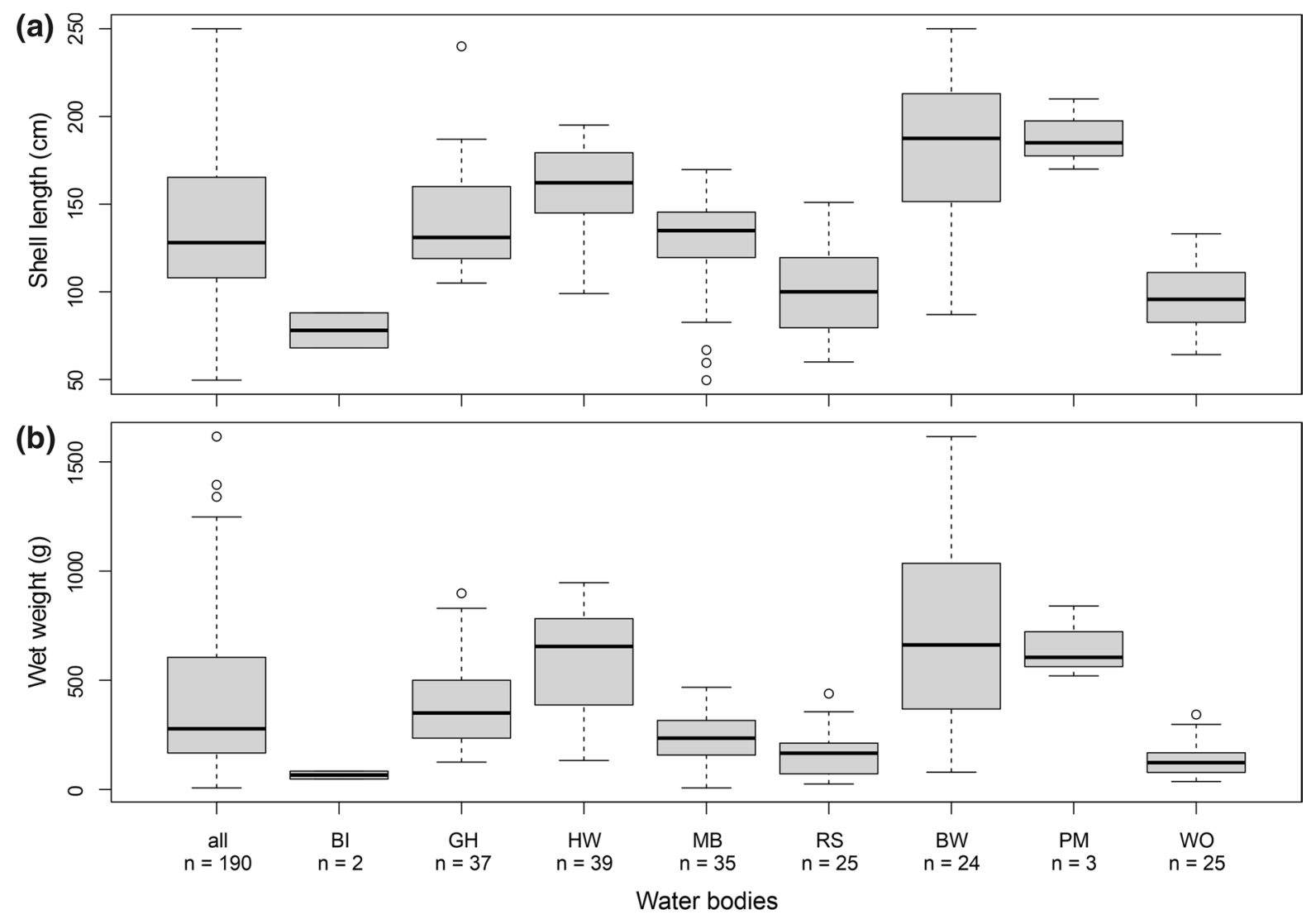

Fig. 3 Boxplots of individual (a) shell lengths and (b) wet weights of $S$. woodiana specimens from each sampled water body. The horizontal lines indicate median values, boxes the 25 th to 75 th percentiles, whiskers the lowest and highest values within 1.5 times the values observed in the percentile boxes and black dots single cases exceeding 1.5 times the values observed in the percentile boxes. Abbreviations for water systems originate from Table 1 
Table 1 Population density (Ind $\mathrm{m}^{-2}$ ) of all mussels and for each species separately for each water system separately and in total

\begin{tabular}{|c|c|c|c|c|c|c|c|c|c|}
\hline \multicolumn{2}{|c|}{ Sampled water systems } & \multicolumn{8}{|c|}{ Mussel density } \\
\hline Names & $\begin{array}{l}\text { Abbrevia- } \\
\text { tion }\end{array}$ & All & $\begin{array}{l}\text { S. woodi- } \\
\text { ana* }\end{array}$ & A. anatina & A. cygnea & U. crassus & U. pictorum & U. tumidus & C. fluminea* \\
\hline $\begin{array}{l}\text { Aischgraben/ } \\
\text { Kleine } \\
\text { Weisach }\end{array}$ & $\mathrm{AK}$ & $\begin{array}{c}1.5 \pm 1.7 \\
{[0.0-5.0]}\end{array}$ & $\begin{array}{l}0.0 \pm 0.0 \\
{[0.0-0.0]}\end{array}$ & $\begin{array}{l}0.8 \pm 1.2 \\
\quad[0.0-3.0]\end{array}$ & $\begin{array}{l}0.0 \pm 0.0 \\
\quad[0.0-0.0]\end{array}$ & $\begin{array}{l}0.0 \pm 0.0 \\
\quad[0.0-0.0]\end{array}$ & $\begin{array}{l}0.8 \pm 0.7 \\
\quad[0.0-2.0]\end{array}$ & $\begin{array}{l}0.0 \pm 0.0 \\
\quad[0.0-0.0]\end{array}$ & $\begin{array}{l}0.0 \pm 0.0 \\
{[0.0-0.0]}\end{array}$ \\
\hline $\begin{array}{l}\text { Backwater } \\
\text { Inn }\end{array}$ & BI & $\begin{array}{c}1.3 \pm 1.4 \\
{[0.0-4.0]}\end{array}$ & $\begin{array}{l}0.3 \pm 0.5 \\
\quad[0.0-1.0]\end{array}$ & $\begin{array}{l}0.3 \pm 0.5 \\
{[0.0-1.0]}\end{array}$ & $\begin{array}{l}0.2 \pm 0.4 \\
\quad[0.0-1.0]\end{array}$ & $\begin{array}{l}0.0 \pm 0.0 \\
\quad[0.0-0.0]\end{array}$ & $\begin{array}{l}0.5 \pm 0.5 \\
{[0.0-1.0]}\end{array}$ & $\begin{array}{l}0.0 \pm 0.0 \\
{[0.0-0.0]}\end{array}$ & $\begin{array}{l}0.0 \pm 0.0 \\
\quad[0.0-0.0]\end{array}$ \\
\hline $\begin{array}{l}\text { Burlafinger } \\
\text { Weiher }\end{array}$ & BW & $\begin{array}{l}2.0 \pm 1.2 \\
{[1.0-5.0]}\end{array}$ & $\begin{array}{c}2.0 \pm 1.2 \\
{[1.0-5.0]}\end{array}$ & $\begin{array}{l}0.0 \pm 0.0 \\
{[0.0-0.0]}\end{array}$ & $\begin{array}{l}0.0 \pm 0.0 \\
{[0.0-0.0]}\end{array}$ & $\begin{array}{l}0.0 \pm 0.0 \\
{[0.0-0.0]}\end{array}$ & $\begin{array}{l}0.0 \pm 0.0 \\
{[0.0-0.0]}\end{array}$ & $\begin{array}{l}0.0 \pm 0.0 \\
{[0.0-0.0]}\end{array}$ & $\begin{array}{l}0.0 \pm 0.0 \\
{[0.0-0.0]}\end{array}$ \\
\hline $\begin{array}{l}\text { Großer } \\
\text { Hirsch- } \\
\text { bergweiher }\end{array}$ & GH & $\begin{array}{l}0.3 \pm 0.4 \\
\quad[0.0-1.1]\end{array}$ & $\begin{array}{l}0.1 \pm 0.1 \\
\quad[0.0-0.3]\end{array}$ & $\begin{array}{l}0.0 \pm 0.0 \\
\quad[0.0-0.0]\end{array}$ & $\begin{array}{l}0.3 \pm 0.4 \\
\quad[0.0-1.1]\end{array}$ & $\begin{array}{l}0.0 \pm 0.0 \\
\quad[0.0-0.0]\end{array}$ & $\begin{array}{l}0.0 \pm 0.0 \\
\quad[0.0-0.0]\end{array}$ & $\begin{array}{l}0.0 \pm 0.0 \\
\quad[0.0-0.0]\end{array}$ & $\begin{array}{l}0.0 \pm 0.0 \\
\quad[0.0-0.0]\end{array}$ \\
\hline Hirtenweiher & HW & $\begin{array}{l}0.4 \pm 0.7 \\
{[0.0-1.9]}\end{array}$ & $\begin{array}{l}0.4 \pm 0.6 \\
{[0.0-1.8]}\end{array}$ & $\begin{array}{l}0.0 \pm 0.0 \\
{[0.0-0.0]}\end{array}$ & $\begin{array}{l}0.0 \pm 0.0 \\
{[0.0-0.1]}\end{array}$ & $\begin{array}{l}0.0 \pm 0.0 \\
{[0.0-0.0]}\end{array}$ & $\begin{array}{l}0.0 \pm 0.0 \\
{[0.0-0.0]}\end{array}$ & $\begin{array}{l}0.0 \pm 0.0 \\
{[0.0-0.0]}\end{array}$ & $\begin{array}{l}0.0 \pm 0.0 \\
{[0.0-0.0]}\end{array}$ \\
\hline Moorbach & $\mathrm{MB}$ & $\begin{array}{l}4.9 \pm 4.9 \\
\quad[0.0-14.0]\end{array}$ & $\begin{array}{l}1.4 \pm 1.2 \\
{[0.0-4.0]}\end{array}$ & $\begin{array}{l}0.0 \pm 0.0 \\
{[0.0-0.0]}\end{array}$ & $\begin{array}{l}0.0 \pm 0.0 \\
{[0.0-0.0]}\end{array}$ & $\begin{array}{l}0.0 \pm 0.0 \\
{[0.0-0.0]}\end{array}$ & $\begin{array}{l}2.3 \pm 2.7 \\
{[0.0-8.0]}\end{array}$ & $\begin{array}{l}1.1 \pm 1.7 \\
{[0.0-4.0]}\end{array}$ & $\begin{array}{l}0.0 \pm 0.0 \\
\quad[0.0-0.0]\end{array}$ \\
\hline Rothsee & RS & $\begin{array}{l}5.8 \pm 7.1 \\
\quad[0.0-26.0]\end{array}$ & $\begin{array}{l}0.2 \pm 0.5 \\
\quad[0.0-2.0]\end{array}$ & $\begin{array}{l}0.4 \pm 0.5 \\
\quad[0.0-1.0]\end{array}$ & $\begin{array}{l}0.0 \pm 0.2 \\
{[0.0-1.0]}\end{array}$ & $\begin{array}{l}0.0 \pm 0.0 \\
{[0.0-0.0]}\end{array}$ & $\begin{array}{l}3.0 \pm 3.3 \\
\quad[0.0-12.0]\end{array}$ & $\begin{array}{l}0.0 \pm 0.0 \\
{[0.0-0.0]}\end{array}$ & $\begin{array}{l}2.1 \pm 4.0 \\
\quad[0.0-13.0]\end{array}$ \\
\hline $\begin{array}{l}\text { Pond near } \\
\text { Muenchs- } \\
\text { muenster }\end{array}$ & $\mathrm{PM}$ & $\begin{array}{l}0.1 \pm 0.3 \\
\quad[0.0-1.0]\end{array}$ & $\begin{array}{l}0.1 \pm 0.2 \\
\quad[0.0-1.0]\end{array}$ & $\begin{array}{l}0.0 \pm 0.1 \\
\quad[0.0-1.0]\end{array}$ & $\begin{array}{l}0.0 \pm 0.2 \\
\quad[0.0-1.0]\end{array}$ & $\begin{array}{l}0.0 \pm 0.0 \\
{[0.0-0.0]}\end{array}$ & $\begin{array}{l}0.0 \pm 0.0 \\
{[0.0-0.0]}\end{array}$ & $\begin{array}{l}0.0 \pm 0.0 \\
\quad[0.0-0.0]\end{array}$ & $\begin{array}{l}0.0 \pm 0.0 \\
{[0.0-0.0]}\end{array}$ \\
\hline Woernitz & WO & $\begin{array}{l}3.7 \pm 5.1 \\
\quad[0.0-20.0]\end{array}$ & $\begin{array}{l}1.3 \pm 0.9 \\
{[0.0-3.0]}\end{array}$ & $\begin{array}{l}0.2 \pm 0.4 \\
\quad[0.0-1.0]\end{array}$ & $\begin{array}{l}0.0 \pm 0.0 \\
\quad[0.0-0.0]\end{array}$ & $\begin{array}{l}0.0 \pm 0.0 \\
\quad[0.0-0.0]\end{array}$ & $\begin{array}{l}2.2 \pm 5.2 \\
\quad[0.0-19.0]\end{array}$ & $\begin{array}{l}0.0 \pm 0.0 \\
\quad[0.0-0.0]\end{array}$ & $\begin{array}{l}0.0 \pm 0.0 \\
\quad[0.0-0.0]\end{array}$ \\
\hline All & & $\begin{array}{l}2.2 \pm 4.4 \\
{[0.0-13.0]}\end{array}$ & $\begin{array}{l}0.5 \pm 0.8 \\
\quad[0.0-5.0]\end{array}$ & $\begin{array}{l}0.2 \pm 0.4 \\
\quad[0.0-3.0]\end{array}$ & $\begin{array}{l}0.1 \pm 0.2 \\
\quad[0.0-1.1]\end{array}$ & $\begin{array}{l}0.0 \pm 0.0 \\
\quad[0.0-0.0]\end{array}$ & $\begin{array}{l}1.0 \pm 2.6 \\
{[0.0-19.0]}\end{array}$ & $\begin{array}{l}0.1 \pm 0.5 \\
\quad[0.0-4.0]\end{array}$ & $\begin{array}{l}0.4 \pm 2.0 \\
\quad[0.0-13.0]\end{array}$ \\
\hline
\end{tabular}

Given values are shown with mean \pm standard deviation in the first line and with the range given in brackets in the second line. An “*” indicates an invasive species

The other abiotic parameters were nearly identical in both groups (Table 1).

Within the studied water bodies, there was a great variance in structure and morphology as well as the measured physico-chemical parameters. In terms of oxygen supply, $S$. woodiana was found over a wide range of dissolved oxygen concentration with a minimum of $4.5 \mathrm{mg} \mathrm{L}^{-1}$ in Moorbach and a maximum of $14.9 \mathrm{mg} \mathrm{L}^{-1}$ in the Burlafinger Weiher (Table 2), even though this sampling cannot be considered representative due to seasonal and daily variation. Accordingly, the redox-potential at $10 \mathrm{~cm}$ depth of the interstitial showed a wide range from $-213 \mathrm{mV}$ in the fish pond Hirtenweiher to $450 \mathrm{mV}$ in the reservoir Rothsee (Table 2). The latter was also the only water body with a mean above the threshold of $300 \mathrm{mV}$ with values below indicating anoxic conditions (Schlesinger 1991). In addition, most of the plots had soft substratum as evident from the low means of penetration resistances (e.g. $0.00 \pm 0.00 \mathrm{~kg} \mathrm{~cm}^{-2}$ in the Burlafinger Weiher and $0.01 \pm 0.02 \mathrm{~kg} \mathrm{~cm}^{-2}$ in the Hirtenweiher, Table 2), and high mean amounts of silt (e.g. $25.5 \pm 11.3 \mathrm{~cm}$ in the Hirtenweiher and $60.0 \pm 54.8 \mathrm{~cm}$ in the Wörnitz, Table 2). $S$. woodiana was found to a maximum water depth of $4.0 \mathrm{~m}$ in the Rothsee. Mean conductivity of the water bodies showed a wide range from $270 \pm 3 \mu \mathrm{S} \mathrm{cm}^{-1}$ in the Burlafinger Weiher to $684 \pm 49 \mu \mathrm{S} \mathrm{cm}^{-1}$ in the Wörnitz (Table 2). 
Table 2 Characterisation of the sampled S. woodiana habitats with water and sediment parameters for each water body and in total

\begin{tabular}{|c|c|c|c|c|c|c|c|c|}
\hline Variables & $\begin{array}{l}\text { BW } \\
(\mathrm{N}=9)\end{array}$ & $\begin{array}{l}\mathrm{GH} \\
(\mathrm{N}=6)\end{array}$ & $\begin{array}{l}\mathrm{HW} \\
(\mathrm{N}=6)\end{array}$ & $\begin{array}{l}\mathrm{BI} \\
(\mathrm{N}=2)\end{array}$ & $\begin{array}{l}\mathrm{MB} \\
(\mathrm{N}=7)\end{array}$ & $\begin{array}{l}\mathrm{RS} \\
(\mathrm{N}=6)\end{array}$ & $\begin{array}{l}\text { WO } \\
(\mathrm{N}=12)\end{array}$ & $\begin{array}{l}\text { All } \\
(\mathrm{N}=48)\end{array}$ \\
\hline \multicolumn{9}{|l|}{ Open water } \\
\hline $\begin{array}{c}\mathrm{O}_{2} \mathrm{FW} \\
\left.\mathrm{L}^{-1}\right)\end{array}$ & $\begin{array}{l}13.6 \pm 0.7 \\
{[12.8-14.9]}\end{array}$ & NA & $\begin{array}{l}11.0 \pm 0.7 \\
{[10.3-12.3]}\end{array}$ & $\begin{array}{l}9.5 \pm 0.3 \\
{[9.3-9.7]}\end{array}$ & $\begin{array}{l}6.1 \pm 1.5 \\
{[4.5-7.9]}\end{array}$ & $\begin{array}{l}8.7 \pm 0.4 \\
{[8.0-9.3]}\end{array}$ & $\begin{array}{l}11.1 \pm 2.2 \\
{[8.0-14.2]}\end{array}$ & $\begin{array}{l}10.4 \pm 2.8 \\
{[4.5-14.9]}\end{array}$ \\
\hline T FW $\left({ }^{\circ} \mathrm{C}\right)$ & $\begin{array}{l}20.2 \pm 0.6 \\
\quad[19.6-21.2]\end{array}$ & NA & $\begin{array}{l}11.4 \pm 0.3 \\
{[11.1-11.7]}\end{array}$ & $\begin{array}{l}10.5 \pm 0.0 \\
{[10.5-10.5]}\end{array}$ & $\begin{array}{l}9.2 \pm 0.6 \\
{[8.4-9.7]}\end{array}$ & $\begin{array}{l}19.1 \pm 4.0 \\
{[16.2-24.8]}\end{array}$ & $\begin{array}{l}22.1 \pm 2.3 \\
\quad[18.9-24.2]\end{array}$ & $\begin{array}{l}17.0 \pm 5.6 \\
{[8.4-24.8]}\end{array}$ \\
\hline $\begin{array}{l}\text { Cond FW }(\mu \mathrm{S} \\
\left.\mathrm{cm}^{-1}\right)\end{array}$ & $\begin{array}{l}270 \pm 3 \\
{[265-274]}\end{array}$ & NA & $\begin{array}{l}593 \pm 4 \\
{[587-598]}\end{array}$ & $\begin{array}{l}291 \pm 0 \\
{[291-291]}\end{array}$ & $\begin{array}{l}589 \pm 36 \\
{[543-635]}\end{array}$ & $\begin{array}{l}562 \pm 9 \\
{[550-568]}\end{array}$ & $\begin{array}{l}684 \pm 49 \\
{[614-732]}\end{array}$ & $\begin{array}{l}530 \pm 164 \\
{[265-732]}\end{array}$ \\
\hline $\mathrm{pH} \mathrm{FW}$ & $\begin{array}{l}8.8 \pm 0.1 \\
{[8.7-8.9]}\end{array}$ & NA & $\begin{array}{l}8.4 \pm 0.1 \\
{[8.3-8.5]}\end{array}$ & $\begin{array}{l}8.0 \pm 0.0 \\
{[8.0-8.1]}\end{array}$ & $\begin{array}{l}7.5 \pm 0.1 \\
{[7.5-7.6]}\end{array}$ & $\begin{array}{l}8.0 \pm 0.0 \\
{[8.0-8.1]}\end{array}$ & $\begin{array}{l}8.2 \pm 0.2 \\
{[8.0-8.6]}\end{array}$ & $\begin{array}{l}8.2 \pm 0.4 \\
{[7.5-8.9]}\end{array}$ \\
\hline Eh FW (mV) & $\begin{array}{l}401 \pm 9 \\
{[385-414]}\end{array}$ & NA & $\begin{array}{l}266 \pm 92 \\
{[87-332]}\end{array}$ & $\begin{array}{l}247 \pm 71 \\
{[196-297]}\end{array}$ & $\begin{array}{l}375 \pm 51 \\
{[304-432]}\end{array}$ & $\begin{array}{l}410 \pm 30 \\
\quad[361-436]\end{array}$ & $\begin{array}{l}416 \pm 179 \\
{[-28-670]}\end{array}$ & $\begin{array}{l}375 \pm 118 \\
{[-28-670]}\end{array}$ \\
\hline Turb (NTU) & $\begin{array}{l}7.5 \pm 3.2 \\
{[4.1-14.3]}\end{array}$ & NA & $\begin{array}{c}35.7 \pm 15.1 \\
\quad[22.1-65.3]\end{array}$ & $\begin{array}{l}35.9 \pm 35.5 \\
{[10.8-61.0]}\end{array}$ & $\begin{array}{l}21.5 \pm 8.0 \\
{[7.7-29.3]}\end{array}$ & $\begin{array}{l}4.6 \pm 1.7 \\
{[2.1-7.2]}\end{array}$ & $\begin{array}{c}16.1 \pm 11.6 \\
{[6.9-43.4]}\end{array}$ & $\begin{array}{c}17.3 \pm 14.9 \\
{[2.1-65.3]}\end{array}$ \\
\hline $\mathrm{v} s u\left(\mathrm{~m} \mathrm{~s}^{-1}\right)$ & $\begin{array}{l}0.00 \pm 0.00 \\
{[0.00-0.00]}\end{array}$ & NA & $\begin{array}{l}0.00 \pm 0.00 \\
{[0.00-0.00]}\end{array}$ & $\begin{array}{c}0.00 \pm 0.00 \\
{[0.00-0.00]}\end{array}$ & $\begin{array}{l}0.10 \pm 0.13 \\
{[0.00-0.28]}\end{array}$ & $\begin{array}{l}0.00 \pm 0.00 \\
{[0.00-0.00]}\end{array}$ & $\begin{array}{l}0.05 \pm 0.06 \\
{[0.00-0.16]}\end{array}$ & $\begin{array}{l}0.03 \pm 0.07 \\
{[0.00-0.28]}\end{array}$ \\
\hline $\mathrm{v} \mathrm{mc}\left(\mathrm{m} \mathrm{s}^{-1}\right)$ & $\begin{array}{l}0.00 \pm 0.00 \\
{[0.00-0.00]}\end{array}$ & NA & $\begin{array}{l}0.00 \pm 0.00 \\
{[0.00-0.00]}\end{array}$ & $\begin{array}{l}0.00 \pm 0.00 \\
{[0.00-0.00]}\end{array}$ & $\begin{array}{c}0.10 \pm 0.12 \\
{[0.00-0.29]}\end{array}$ & $\begin{array}{l}0.00 \pm 0.00 \\
{[0.00-0.00]}\end{array}$ & $\begin{array}{l}0.06 \pm 0.06 \\
{[0.00-0.19]}\end{array}$ & $\begin{array}{l}0.03 \pm 0.07 \\
{[0.00-0.29]}\end{array}$ \\
\hline Depth $(\mathrm{cm})$ & $\begin{array}{c}104.1 \pm 16.8 \\
{[65.0-120.0]}\end{array}$ & $\begin{array}{l}30.7 \pm 4.2 \\
\quad[25.0-36.0]\end{array}$ & $\begin{array}{l}44.2 \pm 19.4 \\
\quad[26.0-76.0]\end{array}$ & $\begin{array}{l}89.0 \pm 4.2 \\
{[86.0-92.0]}\end{array}$ & $\begin{array}{l}52.3 \pm 16.8 \\
\quad[18.0-70.0]\end{array}$ & $\begin{array}{l}296.7 \pm 101.3 \\
{[150.0-400.0]}\end{array}$ & $\begin{array}{l}79.8 \pm 23.2 \\
\quad[59.0-135.0]\end{array}$ & $\begin{array}{l}97.2 \pm 88.0 \\
\quad[18.0-400.0]\end{array}$ \\
\hline Width (m) & NA & NA & NA & $\begin{array}{l}29.8 \pm 8.8 \\
{[23.5-36.0]}\end{array}$ & $\begin{array}{l}3.4 \pm 0.5 \\
{[2.5-4.1]}\end{array}$ & NA & $\begin{array}{c}30.9 \pm 13.7 \\
{[14.8-57.9]}\end{array}$ & $\begin{array}{c}21.6 \pm 16.8 \\
{[2.5-57.9]}\end{array}$ \\
\hline \multicolumn{9}{|l|}{ Substrate } \\
\hline $\begin{array}{c}\mathrm{O}_{2} \mathrm{IN}(\mathrm{mg} \\
\left.\mathrm{L}^{-1}\right)\end{array}$ & $\begin{array}{l}0.6 \pm 1.3 \\
{[0.0-3.9]}\end{array}$ & NA & $\begin{array}{l}0.8 \pm 1.2 \\
\quad[0.0-3.0]\end{array}$ & $\begin{array}{l}1.6 \pm 0.1 \\
{[1.5-1.7]}\end{array}$ & $\begin{array}{l}0.5 \pm 0.5 \\
{[0.0-1.5]}\end{array}$ & $\begin{array}{l}3.1 \pm 1.9 \\
\quad[0.4-6.1]\end{array}$ & $\begin{array}{l}1.1 \pm 1.1 \\
{[0.0-3.3]}\end{array}$ & $\begin{array}{l}1.2 \pm 1.4 \\
{[0.0-6.1]}\end{array}$ \\
\hline $\mathrm{T}$ IN $\left({ }^{\circ} \mathrm{C}\right)$ & $\begin{array}{l}21.6 \pm 0.3 \\
{[20.9-21.9]}\end{array}$ & NA & $\begin{array}{l}12.2 \pm 0.2 \\
{[11.9-12.5]}\end{array}$ & $\begin{array}{l}11.4 \pm 0.7 \\
\quad[10.9-11.9]\end{array}$ & $\begin{array}{l}10.0 \pm 0.8 \\
{[9.0-11.4]}\end{array}$ & $\begin{array}{l}19.2 \pm 4.5 \\
{[15.6-25.3]}\end{array}$ & $\begin{array}{l}21.8 \pm 1.9 \\
\quad[19.3-24.4]\end{array}$ & $\begin{array}{l}17.5 \pm 5.3 \\
{[9.0-25.3]}\end{array}$ \\
\hline $\begin{array}{l}\text { Cond IN }(\mu \mathrm{S} \\
\left.\mathrm{cm}^{-1}\right)\end{array}$ & $\begin{array}{l}356 \pm 81 \\
{[265-530]}\end{array}$ & NA & $\begin{array}{l}655 \pm 63 \\
{[594-736]}\end{array}$ & $\begin{array}{l}394 \pm 37 \\
\quad[367-420]\end{array}$ & $\begin{array}{l}882 \pm 227 \\
{[610-1.282]}\end{array}$ & $\begin{array}{l}639 \pm 87 \\
{[536-768]}\end{array}$ & $\begin{array}{l}792 \pm 97 \\
{[704-1.009]}\end{array}$ & $\begin{array}{l}653 \pm 224 \\
{[265-1282]}\end{array}$ \\
\hline $\mathrm{pH}$ IN & $\begin{array}{l}7.0 \pm 0.2 \\
{[6.8-7.3]}\end{array}$ & NA & $\begin{array}{l}7.6 \pm 0.5 \\
{[7.2-8.4]}\end{array}$ & $\begin{array}{l}7.3 \pm 0.1 \\
{[7.3-7.4]}\end{array}$ & $\begin{array}{l}7.0 \pm 0.4 \\
{[6.6-7.8]}\end{array}$ & $\begin{array}{l}7.2 \pm 0.3 \\
{[7.0-7.7]}\end{array}$ & $\begin{array}{l}7.2 \pm 0.3 \\
{[6.8-7.6]}\end{array}$ & $\begin{array}{l}7.2 \pm 0.3 \\
{[6.6-8.4]}\end{array}$ \\
\hline Eh IN (mV) & $\begin{array}{l}296 \pm 80 \\
{[192-413]}\end{array}$ & NA & $\begin{array}{l}49 \pm 140 \\
{[-213-202]}\end{array}$ & $\begin{array}{l}121 \pm 105 \\
{[46-195]}\end{array}$ & $\begin{array}{c}24 \pm 33 \\
{[-5-76]}\end{array}$ & $\begin{array}{l}356 \pm 83 \\
{[252-450]}\end{array}$ & $\begin{array}{l}133 \pm 136 \\
{[-148-280]}\end{array}$ & $\begin{array}{c}169 \pm 157 \\
{[-213-450]}\end{array}$ \\
\hline PR $\left(\mathrm{kg} \mathrm{cm}^{-2}\right)$ & $\begin{array}{l}0.00 \pm 0.00 \\
{[0.00-0.00]}\end{array}$ & NA & $\begin{array}{l}0.01 \pm 0.02 \\
{[0.00-0.05]}\end{array}$ & $\begin{array}{c}0.03 \pm 0.01 \\
{[0.02-0.03]}\end{array}$ & $\begin{array}{l}0.19 \pm 0.18 \\
{[0.01-0.55]}\end{array}$ & $\begin{array}{l}0.18 \pm 0.11 \\
{[0.07-0.38]}\end{array}$ & $\begin{array}{l}0.27 \pm 0.17 \\
{[0.00-0.51]}\end{array}$ & $\begin{array}{l}0.14 \pm 0.16 \\
{[0.00-0.55]}\end{array}$ \\
\hline Silt layer $(\mathrm{cm})$ & $\begin{array}{c}18.8 \pm 10.1 \\
{[8.0-40.0]}\end{array}$ & $\begin{array}{l}1.7 \pm 2.6 \\
{[0.0-5.0]}\end{array}$ & $\begin{array}{l}25.5 \pm 11.3 \\
\quad[10.0-38.0]\end{array}$ & $\begin{array}{l}6.5 \pm 4.9 \\
{[3.0-10.0]}\end{array}$ & $\begin{array}{l}23.1 \pm 35.4 \\
{[0.0-100.0]}\end{array}$ & NA & $\begin{array}{l}60.0 \pm 54.8 \\
{[0.0-100.0]}\end{array}$ & $\begin{array}{l}20.7 \pm 29.6 \\
{[0.0-100.0]}\end{array}$ \\
\hline Macroph. (\%) & $\begin{array}{r}25.0 \pm 27.4 \\
{[0.0-80.0]}\end{array}$ & $\begin{array}{l}0.0 \pm 0.0 \\
{[0.0-0.0]}\end{array}$ & $\begin{array}{l}0.0 \pm 0.0 \\
{[0.0-0.0]}\end{array}$ & $\begin{array}{r}12.5 \pm 10.6 \\
{[5.0-20.0]}\end{array}$ & $\begin{array}{l}0.0 \pm 0.0 \\
{[0.0-0.0]}\end{array}$ & $\begin{array}{l}0.0 \pm 0.0 \\
{[0.0-0.0]}\end{array}$ & $\begin{array}{l}4.2 \pm 7.9 \\
\quad[0.0-20.0]\end{array}$ & $\begin{array}{l}6.3 \pm 15.4 \\
{[0.0-80.0]}\end{array}$ \\
\hline
\end{tabular}

Given values are shown with mean \pm standard deviation in the first line and with the range given in brackets in the second line. Abbreviations for water systems originate from Table 1

Coexistence of $S$. woodiana with native mussel species

Over all sampling spots, the highest number of individuals over all mussel species $\left(20\right.$ Ind $\left.\mathrm{m}^{-2}\right)$ were found in one spot with $S$. woodiana co-occurring with $U$. pictorum (Fig. 4a.) whereas the highest biomass $\left(3312.0 \mathrm{~g} \mathrm{~m}^{-2}\right)$ was found in one spot with S. woodiana as the only mussel species (Fig. 4b.).
According to the DistLM, the redox of the water column $(\mathrm{AIC}=145.9$, SS-trace $=5870.2$, Pseudo$F=3.0, p<0.05)$ and the temperature of the water column $(\mathrm{AIC}=146.3$, SS-trace $=5205.0$, Pseudo$F=2.6, \quad p<0.05)$ explained $15.0 \%$ and $13.3 \%$, respectively, of the variance of native mussel abundance (Fig. 5a). The redox of the water column $(\mathrm{AIC}=149.2, \quad$ SS-trace $=7337.4, \quad$ Pseudo-F $=3.2$, $p<0.05)$ and conductivity of the interstitial water 

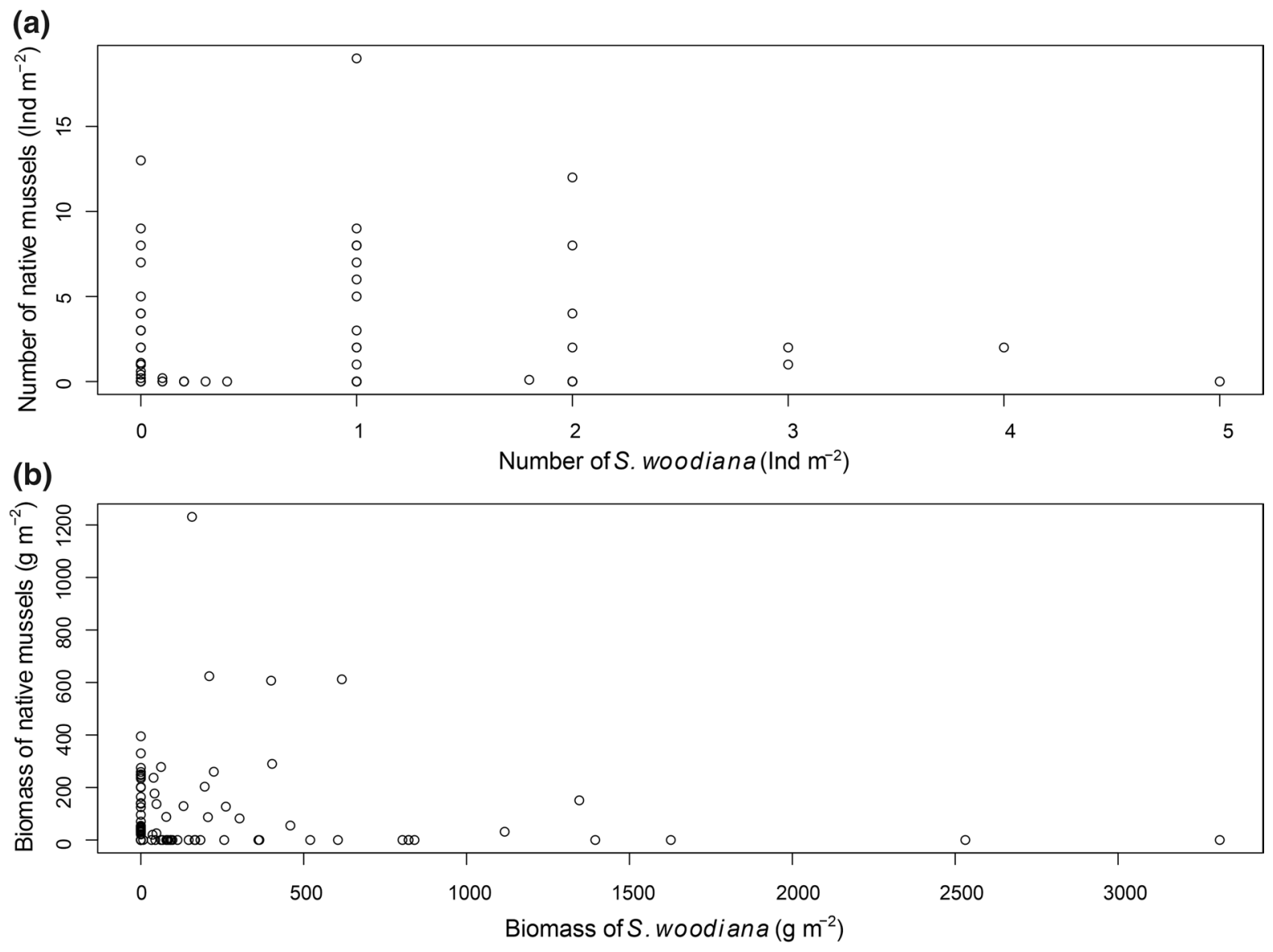

Fig. 4 Total number (a) and biomass (b) of S. woodiana and of native mussel species at each sampling spot

$(\mathrm{AIC}=149.9, \quad$ SS-trace $=5903.5, \quad$ Pseudo- $\mathrm{F}=2.4$, $p<0.05)$ explained $15.6 \%$ and $12.6 \%$, respectively, of the variance of the biomass of native mussels (Fig. 5b). Neither the abundance nor the biomass of $S$. woodiana had a statistically significant contribution to the explanation of the model. Nevertheless, based on the few data points of this study, a high biomass of native mussels was only found in locations with less than $617 \mathrm{~g} \mathrm{~m}^{-2}$ of $S$. woodiana (Fig. 4).

\section{Discussion}

In recent years, there have already been some individual detections of $S$. woodiana in some of the federal states of Germany, e.g. in Baden-Wuertemberg,
Hessen, Saxony, Schleswig-Holstein, and Thuringia (Bahr and Wiese 2018; Bössneck and Klingelhöfer 2011; Dümpelmann 2012; Nagel and Pfeiffer 2019; Pfeiffer 2002). However, all of these studies only documented single populations and did not comprise a systematic sampling approach. In contrast, this study provides the first systematic approach to document the actual distribution of the invasive $S$. woodiana in the federal state of Bavaria in Germany by verifying punctual local information of random observations and anecdotal reporting. Alongside the distribution, we also documented the habitat characteristics as well as the density and biomass of $S$. woodiana and of the co-occurring other native and invasive mussel species. 
Fig. 5 Distance-based redundancy analysis (dbRDA) shows the relation of species composition in density (a) and biomass (b) of native mussels in relation to the physico-chemical variables as well as density or biomass of $S$. woodiana. Bubbles show the number of individuals (a) or biomass (b) per species. Physico-chemical variables shown in a vector overlay have a significant influence on the variability of the species composition and were correlated with the dbRDA plot using Pearson correlation. The length of the lines indicate the strength of correlation, with the radius of the black circle representing $100 \%$ correlation. Vectors indicating the orientation of $S$. woodiana density represent no significant influence and are shown only for illustration
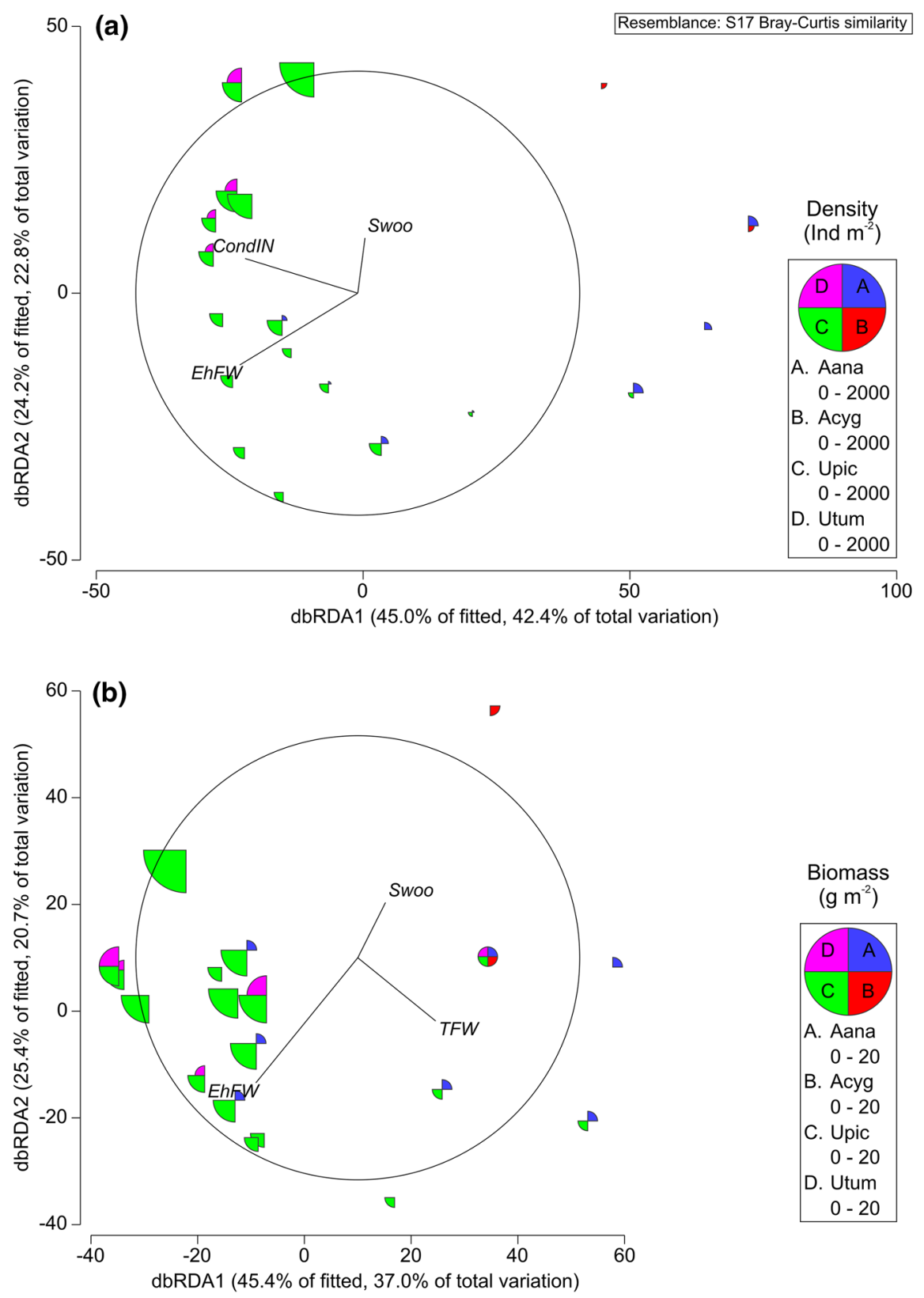

Biomass $\left(\mathrm{g} \mathrm{m}^{-2}\right)$

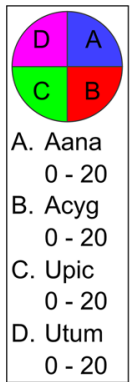

Actual and potential distribution

The examined populations of this study were widespread across Bavaria. Spatial concentrations in some regions were evident, but this may be partly affected by our study approach which focused on a closer inspection of sites with speculated or expected occurrence of $S$. woodiana. Local reporting was strongly dependent on an awareness of the issue of invasive species. Nevertheless, the occurrence of $S$. woodiana was not limited to a special region or drainage system, which suggests that there may be many still undetected populations across Bavaria. It also suggests a greater than expected invasion potential of the species and a limited possibility of still being able to eradicate single 
hotspot populations. This is also obvious from other countries where new populations of $S$. woodiana are frequently found (e.g. Cilenti 2019; Urbańska and Andrzejewski 2019).

Aside from the absent regional limitation, we also found a broad range of suitable habitats, extending from rivers to eutrophic ponds (Table 2) with a great variety of habitat conditions. Our results suggest that many types of inland waters are potentially suitable for $S$. woodiana, which has already been shown by others (e.g. Benkő-Kiss et al. 2013; Douda et al. 2012; Popa 2007; Urbańska and Andrzejewski 2019; Urbańska et al. 2021). S. woodiana is often characterised as a thermophilic species since this mussel originates from tropical and subtropical areas (Kondakov et al. 2018) and has predominantly invaded warmer natural waters in the southern part of Europe (Lajtner and Crncan 2011) as well as artificially heated waters and fish ponds in the northern part (Kraszewski and Zdanowski 2007). Nevertheless, this species has demonstrated an adaption to colder temperatures, which facilitated its spread across Europe (Konecny et al. 2018). This is confirmed by our results. The water temperature was comparatively low in the Moorbach brook and yet we found the smallest individual of S. woodiana in it, indicating that this species is well established and reproducing (Table 3).

Potential pathways

The water usage in many of the studied waters leads to the suggestion that the first spread of $S$. woodiana in Bavaria was limited to waters with artificial stocking. This may be traced back to stockings with grass carp, Ctenopharyngodon idella (Valenciennes 1844), since this fish species is often used to reduce macrophytes within fish ponds (e.g. Pípalová 2006) and is known as a suitable host for $S$. woodiana (Huber and Geist 2019). Most of the pond owners confirmed that they have stocked C. idella and some of them still do. Since these stocked fish are often imported from hatcheries in Poland and Hungary, it is highly likely that alongside the fish, S. woodiana is also introduced attached to the gills of the fish.

Nevertheless, the occurrence of $S$. woodiana is not only limited to fish ponds. In the reservoir Rothsee the main population of this mussel was mainly found in the area next to the in- and outflow of the reservoir that is connected with the Main-Danubechannel. It can be assumed that $S$. woodiana invaded this reservoir from the channel via infected fish. Since $S$. woodiana has already been found in lower parts of the Danube system (e.g. Lajtner and Crncan 2011; Paunovic 2006; Popa 2007), it cannot be excluded that this species has already spread throughout many places connected to the Danube drainage including the Main system. Such artificial connections of naturally separated drainage systems may work as superspreader highways for invasive species and consequently complicate effective management.

Another pathway could be the transfer of infested fish from ponds with an occurrence of $S$. woodiana to uninhabited waters. This seems a general practice in German carp production. Besides common carp, fish farmers usually cultivate secondary species like pike (Esox lucius), perch (Perca fluviatilis), pike-perch (Sander lucioperca) and tench (Tinca tinca) which are often used for re-stocking by fishing clubs (Brämick 2019). To prevent the spread of $S$. woodiana, pond owners and fishing clubs should be
Table 3 Average shell length and wet weight of all $S$. woodiana specimens of each sampled water body and in total including specimens found outside of the plots

\begin{tabular}{lrll}
\hline Water bodies & $\mathrm{n}$ & Average length $\pm \mathrm{SD}(\mathrm{mm})$ & Average weight $\pm \mathrm{SD}(\mathrm{g})$ \\
\hline AK & 0 & - & - \\
BI & 2 & $78.0 \pm 14.1[68.0-88.0]$ & $66.0 \pm 25.5[48.0-84.0]$ \\
BW & 24 & $180.0 \pm 41.3[87.0-250.0]$ & $721.9 \pm 429.7[79.0-1616.0]$ \\
GH & 37 & $143.3 \pm 30.3[105.0-240.0]$ & $402.6 \pm 206.4[125.2-898.2]$ \\
HW & 39 & $157.8 \pm 27.8[99.0-195.1]$ & $581.8 \pm 244.8[133.0-947.0]$ \\
MB & 35 & $127.6 \pm 31.0[49.6-169.7]$ & $236.3 \pm 125.3[7.0-468.0]$ \\
RS & 25 & $100.4 \pm 25.2[60.0-151.0]$ & $161.4 \pm 100.1[25.0-439.0]$ \\
PM & 3 & $188.3 \pm 20.2[170.0-210.0]$ & $655.0 \pm 165.8[520.0-840.0]$ \\
WO & 25 & $96.2 \pm 18.8[64.2-133.1]$ & $138.2 \pm 79.0[36.4-343.0]$ \\
All & 190 & $135.8 \pm 40.9[49.6-250.0]$ & $383.0 \pm 302.1[7.0-1616.0]$ \\
\hline
\end{tabular}


sensitised, in particular since high infestation densities of $S$. woodiana glochidia impair fish physiology and condition (Douda et al. 2017), and, additionally, legal regulations should be adopted to prohibit the transfer of fish from waters with $S$. woodiana populations to natural water bodies. Other possibilities whose implementation would be difficult to control could be to check each fish for glochidia, before transfer, or caging the fish in basins without $S$. woodiana for a time span long enough for glochidia to develop and drop off the fish. Due to the continuous reproduction, a simple regulation linked to timespans without $S$. woodiana glochidia production cannot be recommended (Labecka and Domagala 2018).

\section{Coexistence with native mussels}

Besides S. woodiana populations, we also documented co-occurring mussel species. In almost all sampled water bodies, $S$. woodiana co-occurs with other mussel species, although we only found this in $14.2 \%$ of the sampling spots. It is still not fully understood how the long-term effects of the invasion of $S$. woodiana will affect the native mussel populations but, nevertheless, shifts in the mussel community are already being reported (Urbańska et al. 2019). One sign of $S$. woodiana outcompeting native mussels could be a much higher ratio of fresh empty shells to live specimens of native mussels when compared with $S$. woodiana. For the water bodies we have investigated in our study, it is not possible to explain a potential shift caused or intensified by $S$. woodiana based on this ratio. We found ponds exclusively inhabited by $S$. woodiana with not a single empty shell of native mussel species but with the largest individuals of $S$. woodiana (Burlafinger Weiher). On the other hand, such a ratio was clearly visible in some plots of the river Wörnitz. However, in this case, we had the phenomenon of significantly disturbed shell formation resulting in thinner and easily breakable shells affecting both native and invasive mussel species. Derived from this, the reason for an increased die-off of native mussels cannot only be explained by the invasion of S. woodiana but it might also demonstrate a higher tolerance of $S$. woodiana to some kind of unsuitable habitat conditions. Such a higher tolerance could be an aspect favouring $S$. woodiana in a potential competitive development. As our results show, a negative link between the biomass of $S$. woodiana and other mussel species could be observed. At spots with high $S$. woodiana biomass, a lower biomass of other mussels could be found and vice versa. This suggests that some kind of limitation in the carrying capacity of a water system has been reached. With a faster growth rate (Sárkány-Kiss et al. 2000), S. woodiana could quickly reach a high biomass which, combined with higher filtration rates, leads to an advantage competing for food sources against native mussels (Douda and Čadková 2017). In this way, the slower growing native mussels could be outcompeted as they are deprived of their nutrition. However, in the fish pond Großer Hirschbergweiher a predominant part was covered with a large layer of quite soft silt with only the slower growing and lighter A. cygnea occurring in these areas. The colonisation of $S$. woodiana in this pond was limited to areas with a more sandy substratum. It seems that the higher weight of $S$. woodiana prevented this mussel from gaining a foothold on this fine sediment.

However, S. woodiana shows many other competitive ecological advantages over native mussel species, especially in the most crucial life stage of freshwater mussels. Thus, it could be shown that the viability of glochidia of $S$. woodiana is less affected by higher temperatures than is the case for glochidia of native mussels (Benedict and Geist 2021). This could be an important aspect in times of climate change resulting in an increase in water temperatures. Combined with more frequent breeding, higher amounts of glochidia (Labecka and Czarnoleski 2019; Labecka and Domagala 2018), higher infestation rates, faster development and a broader range of suitable host fish (Douda et al. 2012; Huber and Geist 2019), this all could lead to S. woodiana outcompeting native mussel species. In order to clarify these processes and to document a possible shift in the mussel community, this study should be repeated after several years.

Implication for management

To prevent the endangered native mussel species from the negative impacts of the invasive $S$. woodiana a good management should, inter alia, be based on three strategies: prevention (avoiding the introduction of invasive species by national or international 
regulations as well as by controls at entry points), early detection and rapid eradication (detecting invasives at an early stage of invasion and rapid removal of these species completely and permanently) as well as long-term management (controlling or containing populations of invasives as well as minimising their impacts) (Robertson et al. 2020). In addition to scientific knowledge, a political regulation is imperative for effective management.

With Regulation (EU) 1143/2014, the European Commission implemented a legal basis for preventing and managing the introduction and spread of IAS in all member states. In addition to this regulation, the European Union implemented a regularly updated list of invasive alien species of concern in the Union known as the black list of invasive alien species. For listed species, the member states are urged to make every effort to prevent an introduction of, and to control or eradicate these species and thus, include all the above mentioned strategies. This implies for example regulations on import, trading, transportation or releasing of IAS. Since the invasion of S. woodiana does not stop at borders, it is important that measures to stop further spreading as well as to eradicate this species should be taken transnationally. Furthermore, $S$. woodiana is already established in many European countries and thus, this is not just a challenge for a single country. Nevertheless, the legal regulations and thus, opportunities seem to be quite different. In Poland for example, this species has been listed as invasive since 2012, which has had significant effects on trade in this species (see Urbańska et al. 2019). This is in contrast to Germany, where S. woodiana is still listed as potentially invasive with the need for further investigations. For this reason, we highly recommend the inclusion of $S$. woodiana on this black list because this species fulfills all of the listed criteria of article 4 paragraph 3 of the regulation:

(criterion a) $S$. woodiana is an alien species to the territory of the European Union (see Kondakov et al. 2018; Sousa et al. 2014); (criterion b) the capability of establishing viable populations across many European countries is very well documented (e.g. Benkő-Kiss et al. 2013; Kamburska et al. 2013; Lajtner and Crncan 2011; Munjiu et al. 2020; Paunovic 2006; Urbańska et al. 2019). Our study provides further evidence in favour of adding Germany to the list of countries with widely established $S$. woodiana populations; (criterion c) economical damage could be caused in fish hatcheries by glochidial infestation by $S$. woodiana which can reduce the body mass or condition factor of infected fish (Douda et al. 2017). As described above, many ecological advantages in respect of reproduction are already known (Benedict and Geist 2021; Huber and Geist 2019; Labecka and Czarnoleski 2019; Labecka and Domagala 2018) which can have significant adverse impacts on freshwater mussel biodiversity; (criterion d). The findings of our study combined with all the studies mentioned above highlight the requirement of measures at Union level to prevent a further introduction, establishment or spread (criterion e); as our study shows, the main cause for further spreading of $S$. woodiana can be traced back to fish stockings. Inclusion on the Union list would create a basis on which regulations and procedures could contribute to the implementation of measures in a more targeted and rapid manner to reduce further spreading.

Since it is practically impossible to regularly monitor all waters for $S$. woodiana and other invasive species, early detection and knowledge on the habitat preferences of the species as demonstrated in this study is essential to be able to assess the impact on native mussels. Thus, it helps to understand the processes that go hand in hand with the invasion, and it is crucial to prevent further spreading. Moreover, measures to combat those invasive species should be adopted quickly to prevent a threat for native mussel populations. In addition, public awareness should be increased concerning the identification and spread of invasive mussels. This requires considering the sale of mussels, fish stocking as well as fisheries and pond management.

Acknowledgements We would like to thank A Benedict, S Rüegg, H Imhof, B Panthalil and M Ozgo for their help during fieldwork. We are very grateful for the financial support of the Bavarian State Office for the Environment (LfU) and thank all local environmental protection agencies, local owners of fisheries rights and fisheries authorities for their permissions to conduct this study. We would also like to thank the TUM Graduate School's Department Graduate Center Weihenstephan at the Technische Universitaet Muenchen for their support. The authors thank the two anonymous reviewers for their careful reading of our manuscript and their helpful suggestions.

Authors' contributions All authors contributed to the study conception and design. Material preparation, data collection and analysis were performed by Andreas H. Dobler and Philipp Hoos. The first draft of the manuscript was written by Andreas 
H. Dobler under supervision of Juergen Geist. All authors read and approved the final manuscript.

Funding Open Access funding enabled and organized by Projekt DEAL. This study was funded by the Bavarian State Office for the Environment (LfU).

Availability of data and material Data are available from the authors upon reasonable request.

Code availability Not applicable.

\section{Declarations}

Conflict of interest The authors declare no conflicts of interest.

Ethics approval Since this study only involved freshwater invertebrates (mussels), no ethics approval was required. With respect to species protection and nature conservation legislation, all samplings were reviewed and approved by the District Governments of Middle Franconia (reference number: RMF-SG55.1-8646-7-111-2) of Swabia (reference number: 55.3-8646-2/856) and of Upper Palatinate (reference number: ROP-SG55.1-8622.1-47-2-3) as well as by the administrative district of Passau (reference number: 41.0.03/8231).

Open Access This article is licensed under a Creative Commons Attribution 4.0 International License, which permits use, sharing, adaptation, distribution and reproduction in any medium or format, as long as you give appropriate credit to the original author(s) and the source, provide a link to the Creative Commons licence, and indicate if changes were made. The images or other third party material in this article are included in the article's Creative Commons licence, unless indicated otherwise in a credit line to the material. If material is not included in the article's Creative Commons licence and your intended use is not permitted by statutory regulation or exceeds the permitted use, you will need to obtain permission directly from the copyright holder. To view a copy of this licence, visit http://creativecommons.org/licenses/by/4.0/.

\section{References}

Adam B (2010) L'Anodonte chinoise Sinanodonta woodiana (Lea, 1834) (Mollusca, Bivalvia, Unionidae): une espèce introduite qui colonise le bassin Rhône-Méditerranée. MalaCo 6:278-287

Anderson M, Gorley R, Clarke K (2008) PERMANOVA+ for PRIMER: guide to software and statistical methods. Primer-e, Plymouth, UK

Bahr AL, Wiese V (2018) Freilandvorkommen von Sinanodonta woodiana (LEA 1834) in Ostholstein und Trockenfallen eines Teiches mit umfangreichen Verlusten von Großmuscheln (Bivalvia: Unionidae). Schr Malakozool 30:39-44
Benedict A, Geist J (2021) Effects of water temperature on glochidium viability of Unio crassus and Sinanodonta woodiana: implications for conservation, management and captive breeding. J Molluscan Stud. https://doi.org/10.1093/ mollus/eyab011

Benkő-Kiss Á, Ferincz Á, Kováts N, Paulovits G (2013) Spread and distribution pattern of Sinanodonta woodiana in Lake Balaton. Knowl Manag Aquat Ecosyst. https://doi. org/10.1051/kmae/2013043

Bespalaya YV, Bolotov IN, Aksenova OV, Gofarov MY, Kondakov AV, Vikhrev IV, Vinarski MV (2018) DNA barcoding reveals invasion of two cryptic Sinanodonta mussel species (Bivalvia: Unionidae) into the largest Siberian river. Limnologica 69:94-102. https://doi.org/10. 1016/j.limno.2017.11.009

Bogan AE, Bowers-Altman J, Raley ME (2011) The first confirmed record of the Chinese Pond Mussel (Sinanodonta woodiana) (Bivalvia: Unionidae) in the United States. The Nautilus 125:41-43

Bolotov IN, Bespalaya YV, Gofarov MY, Kondakov AV, Konopleva ES, Vikhrev IV (2016) Spreading of the Chinese pond mussel, Sinanodonta woodiana, across Wallacea: One or more lineages invade tropical islands and Europe. Biochem Syst Ecol 67:58-64. https://doi.org/10.1016/j. bse.2016.05.018

Bössneck U, Klingelhöfer J (2011) Erster Nachweis der Chinesischen Teichmuschel, Sinanodonta woodiana (LEA 1834) aus Thüringen. Mitt Dtsch Malakozool Ges $85: 11-16$

Brämick U (2019) Jahresbericht zur Deutschen Binnenfischerei und Binnenaquakultur 2019.

Cilenti L (2019) First record of Sinanodonta woodiana (Lea, 1834) in an artificial reservoir in the Molise region, Southeast Italy. Bioinvasions Rec 8:320-328. https://doi. org/10.3391/bir.2019.8.2.14

Cohen RRH, Dresler PV, Phillips EJP, Cory RL (1984) The effect of the Asiatic clam, Corbicula fluminea, on phytoplankton of the Potomac River, Maryland. Limnol Oceanogr 29:170-180. https://doi.org/10.4319/lo.1984.29.1. 0170

Cuthbert RN, Pattison Z, Taylor NG et al. (2021) Global economic costs of aquatic invasive alien species. Sci Total Environ:145-238. https://doi.org/10.1016/j.scitotenv. 2021.145238

Dobler AH, Geist J (2022) Impacts of native and invasive crayfish on three native and one invasive freshwater mussel species. Freshw Biol 67(2):389-403. https://doi.org/10. 1111/fwb.13849

Donrovich SW, Douda K, Plechingerová V et al (2017) Invasive Chinese pond mussel Sinanodonta woodiana threatens native mussel reproduction by inducing cross-resistance of host fish. Aquat Conserv 27:1325-1333. https:// doi.org/10.1002/aqc. 2759

Douda K, Čadková Z (2017) Water clearance efficiency indicates potential filter-feeding interactions between invasive Sinanodonta woodiana and native freshwater mussels. Biol Invasions 20:1093-1098. https://doi.org/10.1007/ s10530-017-1615-x

Douda K, Vrtílek M, Slavík O, Reichard M (2012) The role of host specificity in explaining the invasion success of the freshwater mussel Anodonta woodiana in Europe. 
Biol Invasions 14:127-137. https://doi.org/10.1007/ s10530-011-9989-7

Douda K, Velíšek J, Kolářová J, Rylková K, Slavík O, Horký P, Langrová I (2017) Direct impact of invasive bivalve (Sinanodonta woodiana) parasitism on freshwater fish physiology: evidence and implications. Biol Invasions 19:989999. https://doi.org/10.1007/s10530-016-1319-7

Dudgeon D, Arthington AH, Gessner MO et al (2006) Freshwater biodiversity: importance, threats, status and conservation challenges. Biol Rev 81:163-182. https://doi.org/ $10.1017 / \mathrm{s} 1464793105006950$

Dümpelmann C (2012) Erste Freilandnachweise der Chinesischen Teichmuschel Sinanodonta woodiana (Lea) in Hessen mit Anmerkungen zu den Konsequenzen ihrer Verbreitung (Bivalvia: Unionidae). Lauterbornia 74:117-124

Geist J, Auerswald K (2007) Physicochemical stream bed characteristics and recruitment of the freshwater pearl mussel (Margaritifera margaritifera). Freshw Biol 52:2299-2316. https://doi.org/10.1111/j.1365-2427. 2007.01812.x

Huber V, Geist J (2019) Reproduction success of the invasive Sinanodonta woodiana (Lea 1834) in relation to native mussel species. Biol Invasions 21:3451-3465. https://doi. org/10.1007/s10530-019-02060-3

Kamburska L, Lauceri R, Riccardi N (2013) Establishment of a new alien species in Lake Maggiore (Northern Italy): Anodonta (Sinanodonta) woodiana (Lea, 1834) (Bivalvia: Unionidae). Aquat Invasions 8:111-116. https://doi.org/ 10.3391/ai.2013.8.1.13

Karatayev AY, Burlakova LE, Padilla DK (2005) Contrasting Distribution and Impacts of Two Freshwater Exotic Suspension Feeders, Dreissena polymorpha and Corbicula fluminea. In: The Comparative Roles of SuspensionFeeders in Ecosystems. NATO Science Series IV: Earth and Environmental Series. pp 239-262. https://doi.org/10. 1007/1-4020-3030-4_14

Keller RP, Geist J, Jeschke JM, Kühn I (2011) Invasive species in Europe: ecology, status, and policy. Environ Sci Eur 23

Kondakov AV, Bespalaya YV, Vikhrev IV et al (2020) The Asian pond mussels rapidly colonize Russia: successful invasions of two cryptic species to the Volga and Ob rivers. BioInvasions Rec 9:504-518. https://doi.org/10.3391/ bir.2020.9.3.07

Kondakov AV, Palatov DM, Rajabov ZP et al. (2018) DNA analysis of a non-native lineage of Sinanodonta woodiana species complex (Bivalvia: Unionidae) from Middle Asia supports the Chinese origin of the European invaders. Zootaxa 4462:511-522. https://doi.org/10.11646/zootaxa. 4462.4.4

Konecny A, Popa OP, Bartakova V et al (2018) Modelling the invasion history of Sinanodonta woodiana in Europe: Tracking the routes of a sedentary aquatic invader with mobile parasitic larvae. Evol Appl 11:1975-1989. https:// doi.org/10.1111/eva.12700

Kraszewski A, Zdanowski B (2007) Sinanodonta woodiana (Lea, 1834) (Mollusca) - a new mussel species in Poland: occurrence and habitat preferences in a heated lake system. Pol J Ecol 55:337-356

Labecka AM, Czarnoleski M (2019) Patterns of growth, brooding and offspring size in the invasive mussel Sinanodonta woodiana (Lea, 1834) (Bivalvia: Unionidae) from an anthropogenic heat island. Hydrobiologia. https://doi.org/ 10.1007/s10750-019-04141-9

Labecka AM, Domagala J (2018) Continuous reproduction of Sinanodonta woodiana (Lea, 1824) females: an invasive mussel species in a female-biased population. Hydrobiologia 810:57-76. https://doi.org/10.1007/ s10750-016-2835-2

Lajtner J, Crncan P (2011) Distribution of the invasive bivalve Sinanodonta woodiana (Lea, 1834) in Croatia. Aquat Invasions 6:S119-S124. https://doi.org/10.3391/ai.2011.6. $\mathrm{S} 1.027$

LfU (Bayerisches Landesamt für Umwelt) (2020) Artenschutzkartierung Bayern. Augsburg, Germany

Lopes-Lima M, Sousa R, Geist J et al (2017) Conservation status of freshwater mussels in Europe: state of the art and future challenges. Biol Rev 92:572-607. https://doi.org/ 10.1111/brv.12244

Lopes-Lima M, Burlakova LE, Karatayev AY, Mehler K, Seddon M, Sousa R (2018) Conservation of freshwater bivalves at the global scale: diversity, threats and research needs. Hydrobiologia 810:1-14. https://doi.org/10.1007/ s10750-017-3486-7

Lopes-Lima M, Hattori A, Kondo T et al (2020) Freshwater mussels (Bivalvia: Unionidae) from the rising sun (Far East Asia): phylogeny, systematics, and distribution. Mol Phylogenet Evol 146:106755. https://doi.org/10.1016/j. ympev.2020.106755

Lydeard C, Cowie RH, Ponder WF et al (2004) The global decline of nonmarine mollusks. Bioscience 54:321-330. https://doi.org/10.1641/0006-3568(2004)054[0321: tgdonm]2.0.co;2

Malmqvist B, Rundle S (2002) Threats to the running water ecosystems of the world. Environ Conserv 29:134-153. https://doi.org/10.1017/s0376892902000097

Meira A, Lopes-Lima M, Varandas S, Teixeira A, Arenas F, Sousa R (2019) Invasive crayfishes as a threat to freshwater bivalves: Interspecific differences and conservation implications. Sci Total Environ 649:938-948. https://doi. org/10.1016/j.scitotenv.2018.08.341

Munjiu O, Şubernetkii I, Andreev N, Railean N, Toderaș I (2020) Distribution and Characteristics of the Invasive Alien Species Sinanodonta woodiana (Lea, 1834) (Bivalvia: Unionidae) in the Republic of Moldova. Acta Zool Bulg 72:531-538

Nagel K-O, Pfeiffer M (2019) Die Chinesische Teichmuschel (Sinanodonta woodiana) in Baden-Württemberg. [The Chinese pond mussel (Sinanodonta woodiana) in BadenWürttemberg, Germany.].

Ożgo M, Urbańska M, Hoos P et al (2020) Invasive zebra mussel (Dreissena polymorpha) threatens an exceptionally large population of the depressed river mussel (Pseudanodonta complanata) in a postglacial lake. Ecol Evol 10:4918-4927. https://doi.org/10.1002/ece3.6243

Paunovic M (2006) Distribution of Anodonta (Sinanodonta) woodiana (Rea, 1834) in inland waters of Serbia. Aquat Invasions 1:154-160. https://doi.org/10.3391/ai.2006.1.3. 10

Pfeiffer M (2002) Chinesische Teichmuschel, Sinanodonta woodiana (Lea, 1834), nun auch in der Oberlausitz. Ber Naturforsch Ges Oberlausitz 10:67-71 
Phelps HL (1994) The asiatic clam (Corbicula fluminea) invasion and system-level ecological change in the Potomac River Estuary near Washington, D.C. Estuaries 17:614621. https://doi.org/10.2307/1352409

Pípalová I (2006) A Review of Grass Carp Use for Aquatic Weed Control and its Impact on Water Bodies. J Aquat Plant Manage 44:1-12

Popa O (2007) New records of Sinanodonta woodiana (Lea, 1834) (Mollusca: Bivalvia: Unionidae) from Eastern Romania. Aquat Invasions 2:265-267. https://doi.org/10. 3391/ai.2007.2.3.12

R Core Team (2020) R: A language and environment for statistical computing. R Foundation for Statistical Computing. Vienna, Austria

Rabitsch W, Nehring S (2017) Naturschutzfachliche Invasivitätsbewertungen für in Deutschland wild lebende gebietsfremde aquatische Pilze. Niedere Pflanzen Und Wirbellose Tiere. https://doi.org/10.19217/skr458

Regnier C, Fontaine B, Bouchet P (2009) Not knowing, not recording, not listing: numerous unnoticed mollusk extinctions. Conserv Biol 23:1214-1221. https://doi.org/ 10.1111/j.1523-1739.2009.01245.x

Robertson PA, Mill A, Novoa A et al (2020) A proposed unified framework to describe the management of biological invasions. Biol Invasions 22:2633-2645. https://doi.org/ 10.1007/s10530-020-02298-2

Sarkany-Kiss A (1986) Anodonta woodiana (Lea, 1834) a new species in Romania (Bivalvia, Unionacea). Trav Mus Natl Hist Nat Grigore Antipa 28:15-17

Sárkány-Kiss A, Sirbu I, Hulea O (2000) Expansion of the adventive species Anodonta woodiana (Lea, 1834) (Mollusca, Bivalvia, Unionoidea) in central and eastern Europe. Acta Oecol Univ Sibiu 7:49-57

Schlesinger WH (1991) Biogeochemestry. Academic Press, San Diego, CA

Schmidt C, Vandré R (2012) Do signal crayfish Pacifastacus leniusculus harm freshwater pearl mussels? Some field observations. In: Henrikson L, Arvidsson B, Österling M (eds) Aquatic conservation with focus on Margaritifera margaritifera - Proceedings of the International Conference in Sundsvall, Sweden, 12-14 August, 2009. Karlstad University Studies, pp 167-179

Sousa R, Pilotto F, Aldridge DC (2011) Fouling of European freshwater bivalves (Unionidae) by the invasive zebra mussel (Dreissena polymorpha). Freshw Biol 56:867876. https://doi.org/10.1111/j.1365-2427.2010.02532.x

Sousa R, Novais A, Costa R, Strayer DL (2014) Invasive bivalves in fresh waters: impacts from individuals to ecosystems and possible control strategies. Hydrobiologia 735:233-251. https://doi.org/10.1007/s10750-012-1409-1

Sousa R, Nogueira JG, Ferreira A, Carvalho F, Lopes-Lima M, Varandas S, Teixeira A (2019) A tale of shells and claws: The signal crayfish as a threat to the pearl mussel Margaritifera margaritifera in Europe. Sci Total Environ 665:329-337. https://doi.org/10.1016/j.scitotenv.2019.02. 094

Stoeckl K, Geist J (2016) Hydrological and substrate requirements of the thick-shelled river mussel Unio crassus
(Philipsson 1788). Aquat Conserv 26:456-469. https:// doi.org/10.1002/aqc. 2598

Stoeckl K, Denic M, Geist J (2020) Conservation status of two endangered freshwater mussel species in Bavaria, Germany: Habitat quality, threats, and implications for conservation management. Aquat Conserv 30:647-661. https://doi.org/10.1002/aqc.3310

Strayer DL, Dudgeon D (2010) Freshwater biodiversity conservation: recent progress and future challenges. J North Am Benthol Soc 29:344-358. https://doi.org/10.1899/ 08-171.1

Strayer DL, Caraco NF, Cole JJ, Findlay S, Pace ML (1999) Transformation of Freshwater Ecosystems by Bivalves: A case study of zebra mussels in the Hudson River. Bioscience 49:19-27. https://doi.org/10.1525/bisi.1999.49.1.19

Strayer DL, Downing JA, Haag WR, King TL, Layzer JB, Newton TJ, Nichols SJ (2004) Changing perspectives on pearly mussels, North America's most imperiled animals. Bioscience 54:429-439. https://doi.org/10.1641/00063568(2004)054[0429:cpopmn]2.0.co;2

Urbańska M, Andrzejewski W (2019) An invasion in progress-Sinanodonta woodiana (Lea, 1834) (Bivalvia: Unionidae) in Poland. Folia Malacol 27:327-335. https:// doi.org/10.12657/folmal.027.022

Urbańska M, Łakomy A, Andrzejewski W, Mazurkiewicz J (2012) The story of one clam. Probably the oldest location of the Chinese pond mussel Sinanodonta woodiana (Lea, 1834) (Bivalvia, Unionidae) in Poland. Oceanol Hydrobiol Stud. https://doi.org/10.2478/s13545-012-0005-3

Urbańska M, Kirschenstein M, Obolewski K, Ożgo M (2019) Silent invasion: Sinanodonta woodiana successfully reproduces and possibly endangers native mussels in the north of its invasive range in Europe. Int Rev Hydrobiol. https://doi.org/10.1002/iroh.201801971

Urbańska M, Kamocki A, Kirschenstein M, Ożgo M (2021) The Chinese pond mussel Sinanodonta woodiana demographically outperforms European native mussels. Sci Rep. https://doi.org/10.1038/s41598-021-96568-1

Van der Velde G, Rajagopal S, bij de Vaate A (2010) The zebra mussel in Europe. Backhuys/Margraf, Leiden, NL/Weikersheim, DE

Young M, Williams J (1983) The status and conservation of the freshwater pearl mussel Margaritifera margaritifera Linn. in Great Britain. Biol Conserv 25:35-52. https://doi.org/ 10.1016/0006-3207(83)90029-0

Zahner-Meike E, Hanson JM (2001) Effect of muskrat predation on naiads. In: Bauer G, Wächtler K (eds) Ecology and evolution of the freshwater mussels Unionoida. Ecological studies (analysis and synthesis), Vol 145. Springer, Berlin. https://doi.org/10.1007/978-3-642-56869-5_10

Zieritz A, Lopes-Lima M, Bogan AE et al (2016) Factors driving changes in freshwater mussel (Bivalvia, Unionida) diversity and distribution in Peninsular Malaysia. Sci Total Environ 571:1069-1078. https://doi.org/10.1016/j. scitotenv.2016.07.098

Zieritz A, Bogan AE, Froufe E et al (2018a) Diversity, biogeography and conservation of freshwater mussels (Bivalvia: 
Unionida) in East and Southeast Asia. Hydrobiologia 810:29-44. https://doi.org/10.1007/s10750-017-3104-8

Zieritz A, Bogan AE, Rahim KAA et al (2018b) Changes and drivers of freshwater mussel diversity and distribution in northern Borneo. Biol Conserv 219:126-137. https://doi. org/10.1016/j.biocon.2018.01.012
Publisher's Note Springer Nature remains neutral with regard to jurisdictional claims in published maps and institutional affiliations. 